\title{
Performance Analysis of RIS-Assisted Mixed Dual-Hop FSO-RF Communication Systems over Exponentiated Weibull Channels
}

\author{
Xinzhong Liu $\left(\mathbb{D}\right.$, Jian Zou $\mathbb{D}^{\text {, }}$, Wenwu Xie $\mathbb{D}^{D}$, Xin Peng, and Chunlai Li \\ School of Information Science and Engineering, Hunan Institute of Science and Technology, Yueyang 410005, China \\ Correspondence should be addressed to Wenwu Xie; gavinxie@hnist.edu.cn
}

Received 11 July 2021; Accepted 28 September 2021; Published 15 October 2021

Academic Editor: Zhe-Li Liu

Copyright (C) 2021 Xinzhong Liu et al. This is an open access article distributed under the Creative Commons Attribution License, which permits unrestricted use, distribution, and reproduction in any medium, provided the original work is properly cited.

\begin{abstract}
Information security is the basis of economic development, information construction, and the protection of people's and national interest. In this paper, the performance of reconfigurable intelligent surface (RIS)-assisted mixed dual-hop free-space opticalradio frequency (FSO-RF) communication systems is studied and analyzed to ensure the safe transmission of information. In this scheme, it is assumed that the atmospheric turbulence in the fading of the FSO channel follows the exponentiated Weibull distribution model and the fading of the RF channel follows the Rayleigh distribution model. Based on the mathematical function, the probability density function and cumulative distribution function of RIS-assisted mixed dual-hop FSO-RF communication systems are derived. Then, the expression of outage probability and the bit error rate of the system are derived from the channel statistics. Finally, the analysis results are verified by Monte Carlo simulation, and the effects of different parameters on the system performance are analyzed. The simulation results show that the atmospheric turbulence parameters, channel distance of FSO, intensity of atmospheric turbulence, and number of reflectors all affect the system performance.
\end{abstract}

\section{Introduction}

The rapid development of Internet and Internet of Things brings remarkable challenges to information security. It is necessary to take corresponding measures from the aspects of information transmission, information storage, and information interaction to ensure the security of information $[1,2]$. Therefore, the field of information security has attracted the attention of researchers and scholars and has achieved a lot of research results $[3,4]$. Due to its large capacity, high transmission security, strong anti-interference ability, unregulated spectrum, low cost, and flexible installation, free-space optical (FSO) communication plays an increasingly important role in today's communication systems [5]. However, the performance of FSO links is significantly affected by transmission distance, environmental factors, and misalignment between the transmitter and receiver [6]. In [7], the outage performance of the FSO communication system is studied in different environments. The system performance of atmospheric turbulence under different probability distribution models is studied in [8-10].
Experimental verification shows that FSO communication is limited by communication distance, and with the increase in communication distance, the performance of the system will significantly decrease. To expand the communication distance of the FSO communication system, the relaying technology has been introduced, which combines the traditional radio frequency (RF) communication and FSO communication to form a hybrid FSO-RF communication system. In [11-13], the performance of the FSO/RF hybrid communication system under amplification and forwarding (AF) and decoding and forwarding (DF) relay schemes is studied and the results show that the performance of the system mainly depends on the FSO link.

Reconfigurable intelligent surfaces (RISs) are a flat surface integrated by a large number of passive reflector elements. Due to their low power consumption, high communication quality, programmable full band response, simple deployment, and many other advantages, RISs will become one of the key technologies of 6G [14-16]. In [14], the construction principle, operation mechanism, development prospect, and application scenario of RIS are studied 
in detail. In [17-19], with the assistance of RIS technology, the performance of the hybrid FSO/RF communication system has been improved to a certain extent, and with the increase in the reflector components, the performance of the system has also been improved.

To accurately describe the fluctuation of light intensity based on the effective receiving aperture under the turbulence intensity from weak to strong, Barrios et al. proposed the exponential Weibull (EW) fading model suitable for the aperture average condition [20]. However, the outage probability and BER performance of the RISassisted mixed dual-hop FSO-RF communication system based on the EW turbulence distribution model and the influence of pointing error and path loss are rarely reported.

In this paper, for an enterprise scenario, signals are transmitted through the mixed dual-hop FSO-RF system and then reflected to the target users by RIS. Then, we investigate the performance of a mixed dual-hop FSO-RF system based on RIS technology. Considering atmospheric turbulence, pointing error, and path loss, not only the expressions of probability density function (PDF) and cumulative distribution function (CDF) of the channel model are derived but also the closed-form expression of outage probability (OP) and the bit error rate (BER) of the system are given. The simulation results show that with the help of RIS technology, the performance of a mixed dual-hop FSORF system can be effectively improved.

\section{System Model}

In this section, we consider a RIS-assisted mixed dual-hop FSO-RF communication system as shown in Figure 1. A signal source (S) transmits information to the target user (U) with the help of relay node (R) and RIS equipped with $\mathrm{N}$ reflectors, and the DF strategy is adopted. The S-R uses FSO link for communication, and R-RIS and RIS-U communicate with the RF link. First, the RF signal is converted to the FSO signal at $\mathrm{S}$ and data are transmitted to $\mathrm{R}$ through the optical transmitter and optical receiver. Then, the FSO signal is converted to the RF signal at $\mathrm{R}$ and data are transmitted to $\mathrm{U}$ through RIS. In this scheme, it is assumed that the atmospheric turbulence in the fading of the FSO channel follows the EW distribution model and the fading of the RF channel follows the Rayleigh distribution model.

2.1. FSO Transmission Link. According to the design of the system model, the signal at $S$ is given by

$$
S(t)=\sqrt{P_{s}}[\vartheta R(t)+B],
$$

where $P_{s}$ represents the transmitted power, $B$ represents the DC bias, and $\vartheta$ represents the photoelectric conversion coefficient. Then, the signal received at $\mathrm{R}$ can be expressed as

$$
y_{S R}=h_{S R} R_{\theta} A S(t)+n_{S R},
$$

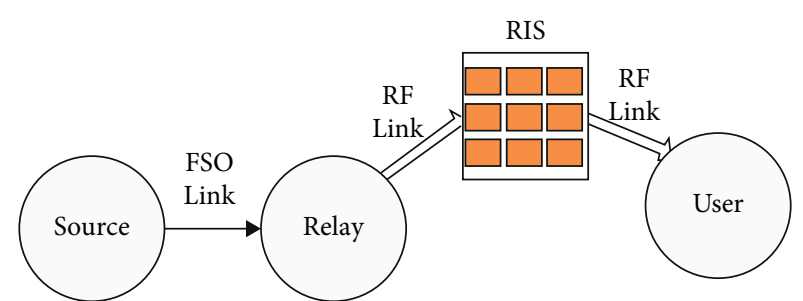

FIGURE 1: Model diagram of the RIS-assisted mixed dual-hop FSORF communication system.

where $R_{\theta}$ and $A$ represent photoelectric response and photoelectric physical area, respectively, and $h_{S R}$ represents random attenuation in the propagation channel. In our model, $h_{S R}$ includes three factors: path loss $h_{l}$, pointing error $h_{p}$, and atmospheric turbulence $h_{a} \cdot n_{S R}$ represents the noise produced by the signal between S and R. In (2), $h_{S R}$ can be obtained as

$$
h_{S R}=h_{l} h_{a} h_{p} .
$$

The path loss caused by the absorption and scattering of the optical signals emitted by the wireless optical communication system satisfies the Beer-Lambert's law:

$$
h_{l}=\exp (-\varsigma l) \text {, }
$$

where $\varsigma$ is the atmospheric attenuation constant and $l$ is the distance of the S-R link. In the Kim model, the atmospheric attenuation constant is defined as $\varsigma=(13 / V)$ $\left(2 \times 10^{7} \times \lambda / 11\right)^{\psi(V)}$, where $\lambda$ represents the wavelength of light, $V$ represents the visible distance, and $\psi(V)$ is a function of visibility given in [21].

EW can describe channel fading under different atmospheric turbulence intensities. Therefore, the PDF can be expressed as [22]

$$
f_{h_{a}}(x)=\frac{m n}{\rho}\left(\frac{x}{\rho}\right)^{n-1} \exp \left(-\left(\frac{x}{\rho}\right)^{n}\right)\left(1-\exp \left(-\left(\frac{x}{\rho}\right)^{n}\right)\right)^{m-1},
$$

where $m, n$, and $\rho$ are parameters related to the scintillation factor and the mean value of light intensity, respectively, and they satisfy $m>0, n>0$, and $\rho>0$ [22].

According to the results in [23], the pointing error obeys Rayleigh distribution, so $h_{p}=A_{0} e^{-2 r^{2} / w^{2 / e}}$, where $r$ is the radial displacement, $A_{0}=\operatorname{erf}^{2}(v), v=\sqrt{\pi / 2} R_{a} / w_{b}$, $\operatorname{erf}($.$) is$ the error function, $R_{a} / w_{b}$ denotes the beam waist normalized by the radius of the receiver aperture, and $w_{e}=\sqrt{\pi} \operatorname{erf}(v) w_{b}^{2} e^{\nu^{2}} / 2 V$ represents the equivalent beam waist. Hence, the PDF can be obtained as

$$
f_{h_{p}}(x)=\frac{\xi^{2}}{A_{0}^{\xi^{\xi}}} x^{\xi^{2}-1}, \quad x \in\left[0, A_{0}\right]
$$

where $\xi=w_{e} / 2 \sigma_{s}$ is the ratio of the beam width to the jitter standard deviation. Using Eq. (4), Eq. (5), and Eq. (6), the joint PDF can be obtained as 


$$
f_{h}(y)=\frac{m n}{\rho^{n}} \frac{\xi^{2}}{\left(h_{l} A_{0}\right)^{\xi^{2}}}(y)^{\xi^{2}-1} \int_{\mathrm{y} /\left(A_{0} h_{l}\right)}^{\infty} x^{n-1-\xi^{2}} \exp \left(-\left(\frac{x}{\rho}\right)^{n}\right)\left(1-\exp \left(-\left(\frac{x}{\rho}\right)^{n}\right)\right)^{m-1} \mathrm{~d} x
$$

According to Newton's general binomial theorem, PDF can be rewritten as

$$
f_{h}(y)=C_{1} y^{\xi^{2}-1} \sum_{j=0}^{\infty} C_{j} \Gamma\left(1-\frac{\xi^{2}}{n}, \frac{1+j}{\left(A_{0} h_{l} \rho\right)^{n}} y^{n}\right),
$$

where $C_{1}=m \xi^{2} /\left(\rho h_{l} A_{0}\right)^{\xi^{2}}$ and $C_{j}=(-1)^{j} \Gamma(m) / \Gamma(m-j)$ $j !(1+j)^{\xi^{2} / n-1}$.

At $\mathrm{R}$, we need to convert an optical signal into an electrical signal through a photoelectric converter, and we can obtain

$$
y_{S R}^{\prime}=\eta h_{S R} R_{\theta} A \sqrt{P_{s}} \varsigma R(t)+n_{1}(t)
$$

where $\eta$ represents the photoelectric conversion efficiency. Hence,

$$
\gamma_{S R}=\bar{\gamma}_{S R} h_{a}^{2} h_{p}^{2}
$$

where $\bar{\gamma}_{S R}=\eta^{2} h_{l}^{2} R_{\theta}^{2} A^{2} P_{s} \varsigma^{2} / N_{1}$. Here, $\gamma_{S R}$ represents the instantaneous signal-to-noise ratio. Under the assumptions of atmospheric turbulence, pointing error, and path loss, the PDF of $\gamma_{S R}$ is given by

$$
f_{\gamma_{S R}}(\gamma)=B_{1} \gamma^{\xi^{2}-2 / 2} \sum_{j=0}^{\infty} C_{j} \Gamma\left(B_{2}, B_{j} \gamma^{n / 2}\right)
$$

where $B_{1}=1 / 2 C_{1} \bar{\gamma}_{S R} \xi^{2^{2}}, B_{j}=1+j /\left(A_{0} h_{l} \rho \sqrt{\bar{\gamma}_{S R}}\right)^{n}$, and $B_{2}$ $=1-\xi^{2} / n$. Then, the CDF can be expressed as

$$
F_{\gamma_{S R}}(\gamma)=\frac{2 B_{1}}{n} \sum_{j=0}^{\infty} C_{j} \gamma^{\xi^{2} / 2} G_{2,3}^{2,1}\left(\begin{array}{r}
\left.B_{j} \gamma^{n / 2}\right|_{0, B_{2},-\frac{\xi^{2}}{n}} ^{1-\epsilon, 1} \\
\xi^{2}
\end{array}\right)
$$

2.2. RF Transmission Main Link. We assume $\varphi_{i}$ and $\psi_{i}$ are the phases, $u$ represents the path loss coefficient, $d_{1}$ is the distance of the R-RIS link, and $d_{2}$ is the distance of the RIS-U link. Then, the channel gain of the R-RIS link can be expressed as $h_{i}=d_{1}^{-u / 2} \alpha_{i} e^{-j \varphi_{i}}$, and the channel of gain of the RIS-U link can be expressed as $g_{i}=d_{2}^{-u / 2} \beta_{i} e^{-j \psi_{i}}$. So, the signal received by the user can be expressed as

$$
y_{R D}=\sqrt{P_{R}} \sum_{i=1}^{N} h_{i} v_{i} g_{i} x+n_{R D}
$$

where $P_{R}$ is the average transmitting power at $R$ and $n_{R U}$ represents additive white Gaussian noise. According to the results in [24], $v_{i}=\rho_{i}\left(\phi_{i}\right) e^{j \phi_{i}}$ represents the reflection coefficient of the $i$-th reflector, where $\rho_{i}\left(\phi_{i}\right)=1$. From [24], we assume $\phi_{i}=\varphi_{i}+\psi_{i}$ in order to obtain the maximum signalto-noise ratio. Then, $y_{R D}$ can be rewritten as

$$
\begin{aligned}
y_{R D} & =\sqrt{P_{R}} \sum_{i=1}^{N} \alpha_{i} \beta_{i} x+n_{R D} \\
& =\sqrt{P_{R}} E x+n_{R D} .
\end{aligned}
$$

According to the results in [14], $\alpha_{i} \beta_{i}$ follows the Rayleigh distribution with a mean value of $\pi / 4$ and a variance of $1-\pi^{2} / 16$. Thus, the instantaneous SNR obtained by the user can be written as

$$
\gamma_{R D}=\bar{\gamma}_{R D} E^{2}
$$

where $\bar{\gamma}_{R U}=P_{R}\left(d_{1} d_{2}\right)^{-u} / N_{2}$. The PDF of $\gamma_{R U}$ is given by [21]

$$
f_{\gamma_{R D}}(x)=\frac{1}{2 \sigma^{2}}\left(\frac{x}{\lambda}\right)^{-1 / 4} \exp \left(-\frac{x+\lambda}{2 \sigma^{2}}\right) I_{-1 / 2}\left(\frac{\sqrt{x \lambda}}{\sigma^{2}}\right) .
$$

The CDF can be obtained as

$$
F_{\gamma_{R D}}\left(\gamma_{t h}\right)=1-Q_{1 / 2}\left(\frac{\sqrt{\lambda}}{\sigma}, \frac{\sqrt{\gamma_{t h}}}{\sigma}\right)
$$

where $Q_{c}(a, b)$ is the Marcum $Q$-function [25]. According to the Gaussian $Q$-function, we can obtain

$$
Q_{1 / 2}(a, b)=Q(a+b)+Q(b-a) .
$$

\section{System Performance Analysis}

3.1. Outage Probability. For the communication system, the $\mathrm{OP}$ is one of the important indicators to measure the transmission reliability of the communication system, which can be expressed as the probability that the SNR of the receiver signal is lower than the threshold value of the set SNR. Therefore, the OP of this system can be written as

$$
\begin{aligned}
P_{o} & =\operatorname{Pr}\left(\gamma_{S R}<\gamma_{t h} \text { or } \gamma_{R D}<\gamma_{t h}\right) \\
& =1-\left(1-F_{\gamma_{S R}}\left(\gamma_{t h}\right)\right)\left(1-F_{\gamma_{R D}}\left(\gamma_{t h}\right)\right) .
\end{aligned}
$$

Combining (12), (17), and (19), the OP can be obtained as

$$
P_{o}=1-\left(1-\frac{2 B_{1}}{n} \sum_{j=0}^{\infty} C_{j} \gamma^{\xi^{2} / 2} G_{2,3}^{2,1}\left(B_{j} \gamma^{\left.\frac{n}{2}\right|_{0, B_{2},-\xi^{2} / n} ^{1-\xi^{2} / n, 1}}\right) Q_{1 / 2}\left(\frac{\sqrt{\lambda}}{\sigma}, \frac{\sqrt{\gamma_{t h}}}{\sigma}\right)\right)
$$


3.2. Asymptotic Outage Probability Analysis. In order to observe the influence of different parameters on the system OP performance, it is necessary to carry out an asymptotic analysis. In the case of high SNR, the system outage performance is mainly determined by the FSO link. Hence, we can obtain

$$
\begin{aligned}
P_{o} & =\operatorname{Pr}\left(\gamma_{S R}<\gamma_{t h} \text { or } \gamma_{R D}<\gamma_{t h}\right) \approx F_{\gamma_{S R}}\left(\gamma_{t h}\right) \\
& =\frac{2 B_{1}}{n} \sum_{j=0}^{\infty} C_{j} \gamma_{t h}^{\xi^{2} / 2} G_{2,3}^{2,1}\left(\begin{array}{c}
n^{1-\xi^{2} / n, 1} \\
\left.B_{j} \gamma_{t h}^{2}\right|_{0, B_{2},-\xi^{2} / n}
\end{array}\right) .
\end{aligned}
$$

According to [26], we have

$$
P_{o}=\frac{1}{n} C_{1} \sum_{j=0}^{\infty} C_{j} \sum_{k=1}^{2} \frac{\prod_{i=1, i \neq k}^{2} \Gamma\left(b_{i}-b_{k}\right) \Gamma\left(b_{k}+\xi^{2} / n\right)}{\Gamma\left(1-b_{k}\right) \Gamma\left(1+\xi^{2} / n+b_{k}\right)\left(\frac{1+j}{\left(A_{0} h_{l} \rho\right)^{n}}\right)^{b_{k}}\left(\frac{\gamma_{t h}}{\bar{\gamma}_{S R}}\right)^{n b_{k} / 2+\xi^{2} / 2},}
$$

where $b_{k} \in\left(0, B_{2},-\xi^{2} / n\right)$. When $\bar{\gamma}_{S R} \longrightarrow \infty$, the diversity order of the system can be expressed as

$$
\begin{aligned}
d & =-\lim _{\rho \longrightarrow \infty} \frac{\log P_{o}}{\log \rho}=\frac{n}{4} \min \left(b_{k}\right)+\frac{\xi^{2}}{2} \\
& =\frac{n}{4} \min \left(0, B_{2},-\frac{\xi^{2}}{n}\right)+\frac{\xi^{2}}{2} .
\end{aligned}
$$

From (23), the asymptotic outage performance of the system depends on the minimum value of $\left(0, B_{2},-\xi^{2} / n\right)$.

3.3. Exact Bit Error Rate Analysis. BER refers to the percentage of error information in the total amount of information in a data transmission process. In the relay system model designed by us, the bit error rate $P_{E 1}$ generated in the FSO link and the bit error rate $P_{E 2}$ generated in the RF link are mainly considered. According to [22], the BER of the system can be written as

$$
P_{\mathrm{BER}}=P_{E 1}+P_{E 2}-2 P_{E 1} P_{E 2} \text {. }
$$

According to the results of [27], we can obtain

$$
P_{E}=\frac{q^{p}}{2 \Gamma(p)} \int_{0}^{\infty} e^{-q \gamma} \gamma^{p-1} F(\gamma) \mathrm{d} \gamma
$$

The precondition is binary modulation. Combining (12) and (25), $P_{E 1}$ can be written as

$$
P_{E 1}=\frac{B_{1}}{n} \frac{2^{B_{2}-3 / 2} n^{\xi^{2}+1 / 2}}{(2 \pi)^{n / 2}} \sum_{j=0}^{\infty} C_{j 4+n, 6}^{4,2+n}\left(\left.\frac{B_{j}^{2} n^{n}}{4}\right|_{0,1 / 2,1 / 2 B_{2}, 1 / 2\left(B_{2}+1\right),-\xi^{2} / 2 n, 1 / 2\left(1-\xi^{2} / n\right)} ^{-\xi^{2} / 2 n, \ldots, 1 / n\left(n-\xi^{2} / 2-1\right), 1 / 2\left(1-\xi^{2} / n\right), 1 / 2\left(2-\xi^{2} / n\right), 1 / 2,1}\right) .
$$

We will only consider DBPSK modulation here, so $p=1$ and $q=1$. Combining (17) and (25), $P_{E 2}$ can be written as [25]

$$
P_{E 2}=\frac{1}{2 \sqrt{1+2 \sigma^{2}}} e^{-\lambda / 2 \sigma^{2}} F_{1}\left(\frac{1}{2}, \frac{1}{2}, \frac{\lambda}{2 \sigma^{2}+4 \sigma^{4}}\right) .
$$

\section{Numerical Results}

According to the expression of OP and BER derived above, we give the simulation results of the proposed communication system model and mainly analyze the influence of different parameter values on the system OP and BER. For this purpose, the following parameters are assumed: $\lambda=10^{-9} \mathrm{~km}, V=1.2 \mathrm{~km}, R_{\theta}=1, P_{s}=50, \varsigma=0.8 A=0.1$, $d_{1}=2, d_{2}=2, u=2$, and $P_{R}=1$.

Figure 2 demonstrates the influence of SNR threshold and channel distance on system outage probability under different SNRs. It is obvious from Figure 2 that when the SNR threshold is constant, the OP of the system increases with the increase in the channel distance. When the channel distance is constant, the OP of the system increases with the increase in the SNR threshold. In other words, the setting of SNR threshold and channel length has a significant impact on the performance of the communication system.

In Figure 3, the variation curves of the parameters $\xi, m$, $n$, and $\rho$ with the system outage probability under different SNRs are analyzed. According to the curve in the figure, it can be concluded that when the parameters $m, n$, and $\rho$ are 


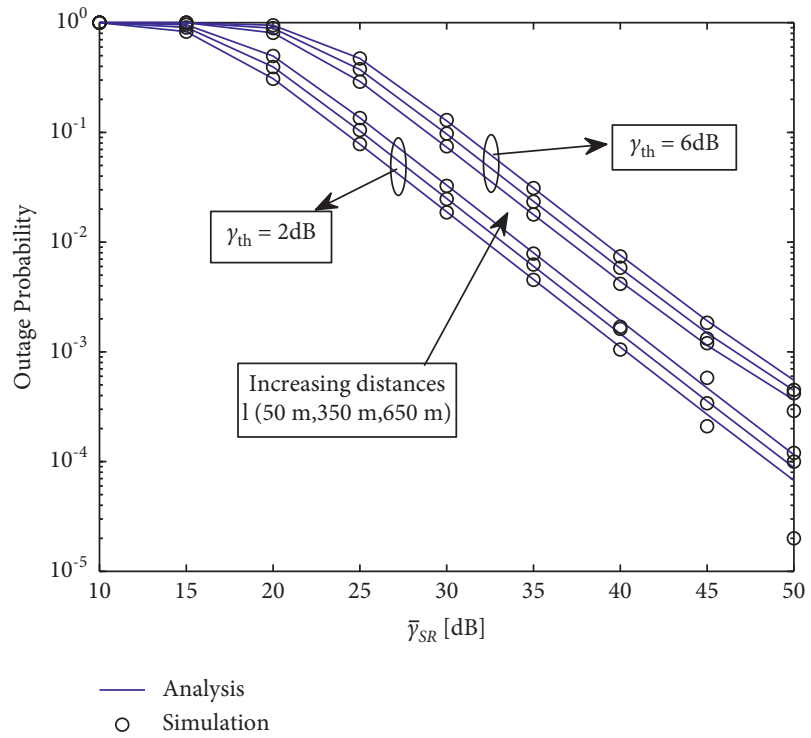

FIGURE 2: OP with different values of SNR threshold and channel distance.

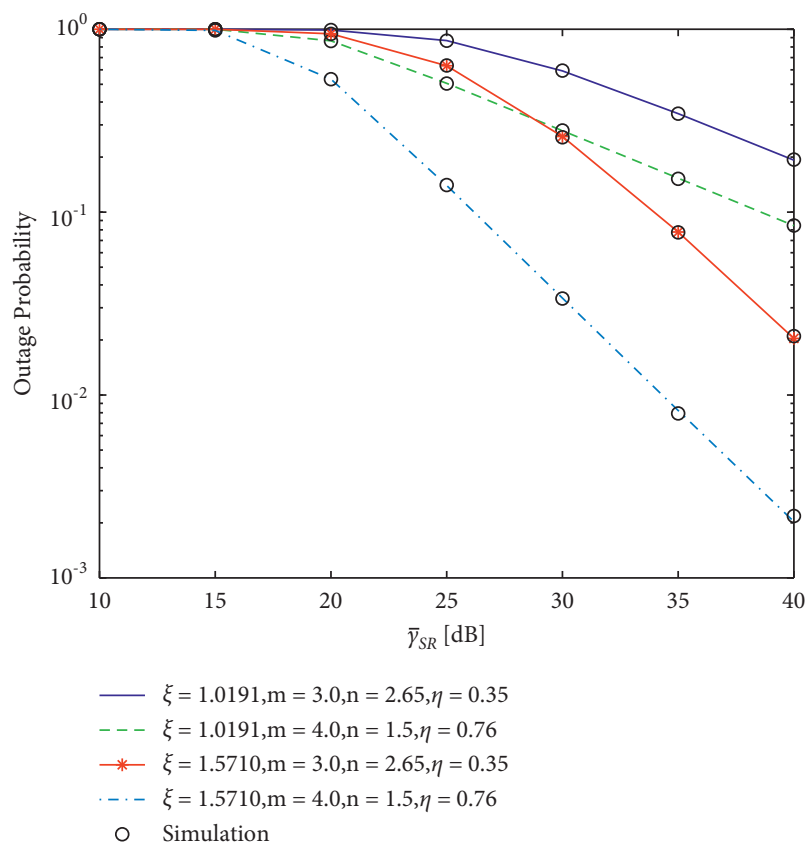

FIgUre 3: OP with different values of parameters $(\xi,(m), n, \rho)$.

constant, the outage probability of the system decreases with the increase in the parameter $\xi$. The reason is that the parameter $\xi$ is inversely proportional to the jitter standard deviation, and the larger the pointing error, the lower the system performance.

Figure 4 illustrates the change in BER under different SNR conditions and the number of reflectors $\mathrm{N}$ for the considered mixed dual-hop FSO-RF-RIS communication system. It can also be seen from Figure 4 that the BER of the system tends to be stable at a high SNR regardless of the value of $N$. As the number of reflectors $\mathrm{N}$ increases, the BER of the system decreases. With the assistance of

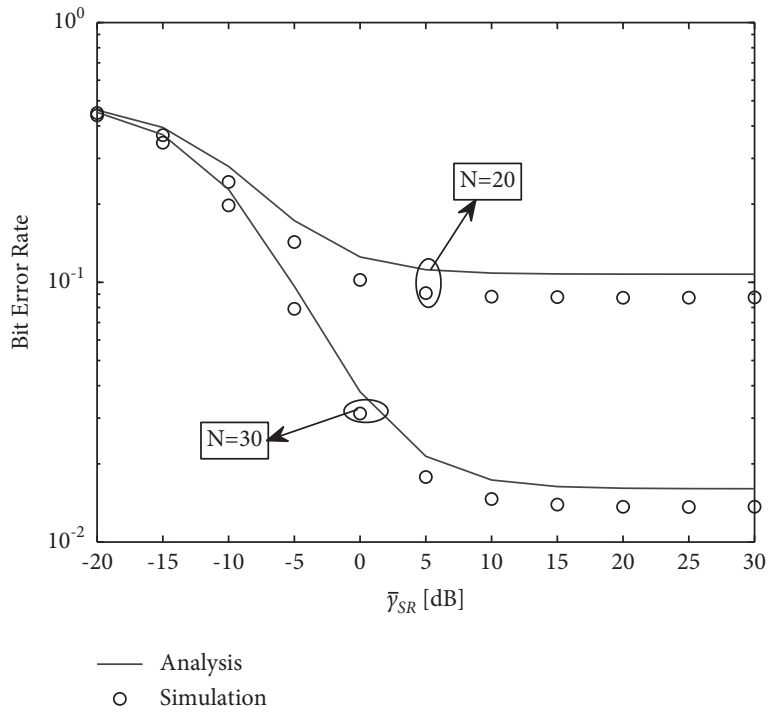

FIGURE 4: BER with a different number of reflectors.

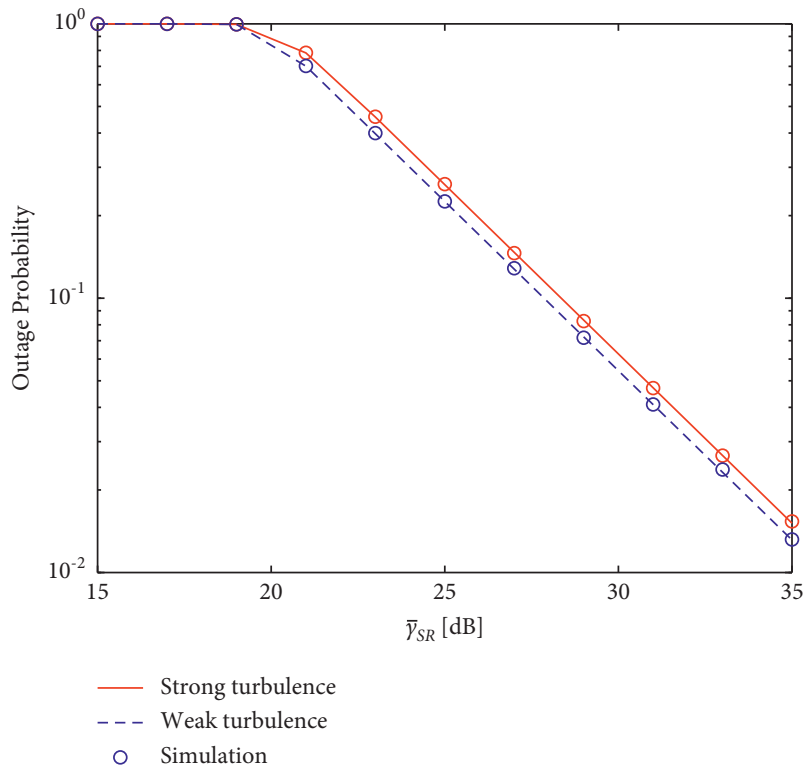

FIGURE 5: OP with different intensities of atmospheric turbulence.

RIS, the BER of the mixed dual-hop FSO-RF relay communication system decreases with the increase in the number of reflectors $\mathrm{N}$.

Figure 5 compares the system outage performance under different SNR conditions and different atmospheric turbulence intensities. According to the simulation results, when the SNR is constant, the OP of the system increases with the increase in the atmospheric turbulence intensity.

\section{Conclusion}

In this paper, the RIS technology is introduced on the basis of the mixed dual-hop FSO-RF relay communication system. Considering pointing error, path loss, and atmospheric 
turbulence, PDF and CDF of the composite channel are derived and the $\mathrm{OP}$ and BER of the communication system are obtained by using mathematical function. The simulation results show that channel distance and SNR threshold are inversely proportional to system performance. The larger the pointing error, the lower the system performance. With the assistance of RIS technology, the BER of the system is significantly reduced with the increase in the number of reflectors $\mathrm{N}$.

\section{Data Availability}

No data were used to support this study.

\section{Conflicts of Interest}

The authors declare that they have no conflicts of interest in this work.

\section{Acknowledgments}

This research was supported by the key scientific research project of Hunan Provincial Department of Education (18A320) and Hunan Natural Science Foundation (2020JJ4342).

\section{References}

[1] S. Nowaczewski and W. Mazurczyk, "Securing future Internet and 5G using customer edge switching using DNSCrypt and DNSSEC," Journal of Wireless Mobile Networks, vol. 11, no. 3, pp. 87-106, 2020.

[2] M. Park, S. Kim, and J. Kim, "Research on note-taking apps with security features," Journal of Wireless Mobile Networks, vol. 11, no. 4, pp. 63-76, 2020.

[3] G. S. Kasturi, A. Jain, and J. Singh, "Detection and classification of radio frequency jamming attacks using machine learning," Journal of Wireless Mobile Networks, vol. 11, no. 4, pp. $49-62,2020$.

[4] A. Abhishta, W. van Heeswijk, M. Junger, L. J. M. Nieuwenhuis, and R. Joosten, "Why would we get attacked? An analysis of attacker's aims behind DDoS attacks," Journal of Wireless Mobile Networks, vol. 11, no. 2, pp. 3-22, 2020.

[5] D. J. T. Heatley, D. R. Wisely, I. Neild, and P. Cochrane, "Optical wireless: the story so far," IEEE Communications Magazine, vol. 36, no. 12, pp. 72-74, 1998.

[6] M. A. Khalighi and M. Uysal, "Survey on free space optical communication: a communication theory perspective," IEEE Commun. Surveys Tuts.vol. 16, no. 4, pp. 2231-2258, 2014.

[7] W. Gappmair, "Further results on the capacity of free-space optical channels in turbulent atmosphere," IET Communications, vol. 5, no. 9, pp. 1262-1267, 2011.

[8] I. S. Ansari, F. Yilmaz, and M. S. Alouini, "Performance analysis of FSO links over unified gamma-gamma turbulence channels," IEEE Vehicular Technology Conference, vol. 2015, 2015.

[9] W. G. Alheadary, K.-H. Park, and M.-S. Alouini, "Performance analysis of multihop heterodyne free-space optical communication over general Malaga turbulence channels with pointing error," Optik, vol. 151, pp. 34-47, 2017.
[10] P. K. Sharma, A. Bansal, P. Garg, T. A. Tsiftsis, and R. Barrios, "Performance of FSO links under exponentiated Weibull turbulence fading with misalignment errors," Nature, vol. 433, p. 7021, 2015.

[11] S. Anees and M. R. Bhatnagar, "Performance evaluation of decode-and-forward dual-hop asymmetric radio frequencyfree space optical communication system," IET Optoelectronics, vol. 9, no. 5, pp. 232-240, 2015.

[12] S. Anees and M. R. Bhatnagar, "Performance of an amplifyand-forward dual-hop asymmetric RF-FSO communication system," Journal of Optical Communications and Networking, vol. 7, no. 2, pp. 124-135, 2015.

[13] L. Kong, W. Xu, L. Hanzo, H. Zhang, and C. Zhao, "Performance of a free-space-optical relay-assisted hybrid RF/FSO system in generalized $\$ \mathrm{M} \$$-distributed channels," IEEE Photonics Journal, vol. 7, no. 5, pp. 1-19, 2015.

[14] E. Basar, M. Di Renzo, J. De Rosny, M. Debbah, M.-S. Alouini, and R. Zhang, "Wireless communications through reconfigurable intelligent surfaces," IEEE Access, vol. 7, pp. 116753-116773, 2019.

[15] E. Basar, "Reconfigurable intelligent surface-based index modulation: a new beyond MIMO paradigm for 6G," IEEE Transactions on Communications, vol. 68, no. 5, pp. 31873196, 2020.

[16] C. Huang, A. Zappone, G. C. Alexandropoulos, M. Debbah, and C. Yuen, "Reconfigurable intelligent surfaces for energy efficiency in wireless communication," IEEE Transactions on Wireless Communications, vol. 18, no. 8, pp. 4157-4170, 2019.

[17] Q. Wu and R. Zhang, "Towards smart and reconfigurable environment: intelligent reflecting surface aided wireless network," IEEE Communications Magazine, vol. 58, no. 1, pp. 106-112, 2020.

[18] L. Yang, W. Guo, and I. S. Ansari, "Mixed dual-hop FSORF communication systems through reconfigurable intelligent surface," IEEE Communications Letters, vol. 99, p. $1,2020$.

[19] A. Sikri, A. Mathur, P. Saxena, M. R. Bhatnagar, and G. Kaddoum, "Reconfigurable intelligent surface for mixed FSO-RF systems with Co-channel interference," IEEE Communications Letters, vol. 99, p. 1, 2021.

[20] R. Barrios and F. Dios, "Exponentiated Weibull distribution family under aperture averaging for Gaussian beam waves," Optics Express, vol. 20, no. 12, pp. 13055-13064, 2012.

[21] I. Kim, B. McArthur, and E. Korevaar, "Comparison of laser beam propagation at $785 \mathrm{~nm}$ and $1550 \mathrm{~nm}$ in fog and haze for optical wireless communications," Optical Wireless Communications III - Proceedings of SPIE, vol. 4214, pp. 26-37, 2001.

[22] R. Barrios and F. Dios, "Exponentiated Weibull model for the irradiance probability density function of a laser beam propagating through atmospheric turbulence," Optics \& Laser Technology, vol. 45, pp. 13-20, 2013.

[23] M. Cheng, Y. Zhang, J. Gao, F. Wang, and F. Zhao, “Average capacity for optical wireless communication systems over exponentiated Weibull distribution non-Kolmogorov turbulent channels," Applied Optics, vol. 53, no. 18, pp. 4011-4017, 2014.

[24] Q. Wu and R. Zhang, "Intelligent reflecting surface enhanced wireless network via joint active and passive b," IEEE Transactions on Wireless Communications, vol. 18, no. 11, pp. 5394-5409, 2019.

[25] V. M. Kapinas, S. K. Mihos, and G. K. Karagiannidis, "On the monotonicity of the generalized Marcum and n," IEEE 
Transactions on Information Theory, vol. 55, no. 8, pp. 37013710, 2009.

[26] Wolfram, "The wolfram functions site," 2001, http:// functions.wolfram.com.

[27] I. S. Ansari, S. Al-Ahmadi, F. Yilmaz, M.-S. Alouini, and H. Yanikomeroglu, "A new formula for the BER of binary modulations with dual-branch selection over generalized-K composite fading channels," IEEE Transactions on Communications, vol. 59, no. 10, pp. 2654-2658, 2011. 